\title{
ASUNTOS LEGALES EN EL
MINISTERIO
}

Norton, Ricardo

Andrews University

ricardo@andrews.edu

Fecha de recepción: Agosto 2013

Fecha de aceptación y versión final: Noviembre 2013

La iglesia debe ser un lugar seguro donde los feligreses son amados y protegidos y es la responsabilidad del ministro velar para que los miembros de su iglesia puedan adorar en paz, en un ambiente seguro donde los derechos morales y civiles de los adoradores son respetados. Este artículo estudia algunos de los asuntos legales más comúnmente asociados con el ministerio, con el fin de informar a los lectores acerca de posibles problemas legales que pueden aflorar en la iglesia local. Sugerencias prácticas y medidas preventivas son sugeridas al final de cada asunto legal discutido, de manera que los dirigentes de iglesia puedan evadir problemas legales, mientras hacen la obra de Dios.

Palabras clave: Invasión de privacidad, difamación de carácter y derechos de autor. 


\section{LEGAL ISSUES IN THE MINISTRY}

Summary: The local church should be a safe place where members feel loved and protected. It is the responsibility of the pastor to ensure a safe environment where moral and civil rights are respected and where church members can worship God in peace. This article studies some of the most common legal issues associated to ministry and informs readers about possible legal problems that may surface in the church context. Preventive measures and practical insights are suggested at the end of the section to help church leaders avoid legal problems while doing God's work.

Keywords: Invasion of privacy, defamation of character, and copyright infringement. 


\section{Introducción}

La naturaleza litigiosa de la sociedad en que vivimos, requiere que los ministros y líderes religiosos se mantengan informados acerca de las leyes y asuntos legales asociados a la iglesia. Contiendas y problemas que en el pasado se solucionaban en la alcoba matrimonial, en el comité de la iglesia o con un simple diálogo con el pastor o el anciano de iglesia; hoy día, son asuntos ventilados en la corte civil. Los ministros deben prestar atención cuidadosa a sus obligaciones legales para prevenir juicios costosos y dolorosos.

Los asuntos legales, discutidos en este artículo, fueron seleccionados tomando en cuenta 38 años de experiencia ministerial del autor, conversaciones con abogados que se especializan en asuntos legales relacionados al ministerio y publicaciones serias relacionadas al tema. Es el deseo del autor que las realidades legales discutidas en este artículo, lejos de producir ansiedad en el ministerio, ayude a los ministros a enfocarse en los derechos y necesidades legales de sus constituyentes y de organizaciones a las que sirven.

\section{Difamación de carácter}

La difamación significa desacreditar a alguien con palabras o por escrito, con el fin de dañar su reputación. ${ }^{1}$ Si la difamación es escrita o mencionada en medios de comunicación pública, se le llama libelo. Este tipo de difamación puede incluir la publicación de fotografías o calumnias. ${ }^{2}$ El libelo puede ser sostenido sin prueba de daños específicos $^{3}$ y generalmente es

1 Diccionario de la lengua española: Real Academia Española, s.v. "Difamar".

2 Richard R. Hammar, Pastor, Church \& Law, 3. ${ }^{a}$ ed. (Matthews, NC: Christian Ministry Resources, 2000). Véase también, Law.com, "Legal Dictionary", Law.com Dictionary, http://dictionary.law.com/ (consultado: 09 de septiembre, 2013). La palabra "libelo", proviene del inglés libel, definido por la RAE como un "Escrito en que se denigra o infama a alguien o algo".

3 Lindell L. Gumper, Legal Issues in the Practice of Ministry (Birmingham, MI Psychological Studies and Consultation 
una acción seguida por personas que buscan dinero, no acción criminal. ${ }^{4}$ En vista a la facilidad con la que una persona o institución puede ser llevada a juicio, por libelo, es importante que se ejerza mucho cuidado con acusaciones o comentarios que puedan hacerse en reuniones oficiales. Debe notarse que la verdad es una defensa absoluta y que no se puede enjuiciar exitosamente a una persona o institución por decir o escribir una verdad. 5 Por otro lado, generalmente no hay protección en contra de reclamos hechos por personas que no son miembros de iglesia o personas que no tienen ninguna obligación constitucional con la institución. ${ }^{6}$

Lindell Gumper asevera que para que un juicio basado en difamación pueda tener éxito, Program, 1981), 21.

4 Mary Angela Shaughnessy, Ministry and the Law: What you Need to Know (New York: Paulist Press, 1998), 29.

5 Clarance E. Hagglund y Britton D. Weimer, Stay Out of Court and Stay in Ministry (Lima, OH: CSS Publishing Company, 1998), 21.

6 Ibíd., 20. la víctima debe demostrar los daños recibidos, tales como perjuicio a su negocio, reputación vocacional o conducta criminal. ${ }^{7}$ La gente que habla con cautela de otras personas debe considerar que la veracidad de una afirmación negativa es la mejor defensa contra los juicios de difamación o libelo.

Aunque el púlpito es el lugar donde el ministro o administrador está más visiblemente expuesto a expresar ideas que lo lleven a ser acusado por difamación, ${ }^{8}$ la difamación de carácter puede darse con mayor frecuencia en las reuniones de la comisión que elige anualmente a los nuevos oficiales de iglesia. ${ }^{9}$ En estas reuniones, es fácil que se mencionen rumores falsos o negativos acerca de una persona de manera que no sea elegida para la posición

7 Gumper, 21.

8 Juicios contra indiscreciones mencionadas desde el púlpito son contundentes cuando las palabras del ministro o administrador son grabadas y usadas en la corte como evidencia.

9 Esta comisión es conocida en la Iglesia Adventista del Séptimo Día, como la "Comisión de Nombramientos". 
propuesta, desafortunadamente, este tipo de difamación con frecuencia llega a oídos de las personas afectadas, proveyendo una base sólida para una acción legal contra la iglesia.

Medidas preventivas. Para protegerse de posibles litigios de difamación, algunas organizaciones religiosas contratan compañías de seguro para protegerse contra juicios basados en negligencia profesional. Sin embargo, tal gasto sería innecesario si los ministros fueran instruidos acerca de las conse-

80 cuencias legales asociadas con acciones y palabras difamadoras durante actividades organizadas por la iglesia. Otra medida preventiva consiste en educar a los miembros de iglesia para no divulgar falsos rumores acerca de otras personas de la iglesia o la comunidad.

\section{Invasión de privacidad}

La corte de justicia permite que la Iglesia sea enjuiciada por "invasión de privacidad" en casos extremos en los que se haya interferido en las relaciones privadas de un individuo o una familia nuclear. ${ }^{10} \mathrm{La}$ invasión de privacidad es definida como la intrusión en la vida personal de otra persona. Algunos autores ${ }^{11}$ aseveran que la invasión de privacidad se manifiesta en cualquiera de las siguientes formas: (1) revelar al público asuntos privados; (2) usar, sin permiso, el nombre o fotografías de otra persona; (3) denigrar públicamente con falsedad a una o más personas; o (4) entrometerse en la vida privada del prójimo.

En la mayoría de los casos, las personalidades públicas no son protegidas de la invasión de privacidad, debido a su posición, influencia y exposición pública ante los medios de comunicación masiva. Por otro

10 Por ejemplo, declarar que un matrimonio no es legítimo porque uno de los cónyuges no es miembro bautizado de la iglesia; a pesar de estar legalmente casado de acuerdo a las leyes civiles de la comunidad. Véase, Hagglund y Weimer, 22.

11 Hammar, 164. Véase también, Thomas F. Taylor, Seven Deadly Lawsuits: How Ministers Can Avoid Litigation and Regulation (Nashville, TN: Abingdon Press, 1996), 121. 
lado, personas que no representan públicamente a la comunidad, tienen más derecho a la privacidad y son protegidas por la ley en casos tales como: (1) Intrusión en su aislamiento y asuntos privados; (2) revelación pública de información privada que pueda avergonzarlos; (3) publicidad falsa y (4) apropiación de su nombre o fotografías con fines lucrativos.

Miembros de Iglesia bien intencionados, con frecuencia sugieren al ministro que visite a familias de la iglesia con problemas que no son de conocimiento general. Sin embargo, la visita pastoral no solicitada a una familia o miembro de iglesia puede ser considerada como invasión de privacidad. No importa cuanto sea apreciado el pastor de la iglesia, hay personas que prefieren que la gente, incluyendo el ministro, se mantenga al margen de los problemas personales y familiares que experimentan. Elena G. de White indicó que dentro de cada familia hay un círculo secreto que debe preservarse y que "Ninguna otra persona tiene el derecho a cruzar ese círculo sagrado". ${ }^{12}$

\section{Medidas preventivas}

Es aconsejable que el ministro se abstenga de dar consejos en asuntos privados a personas que no lo solicitan. Respetar la privacidad del miembro de iglesia y de la familia nuclear es señal de madurez espiritual y de liderazgo efectivo. Taylor recomienda las siguientes medidas preventivas, con el fin de proteger la privacidad de las personas y de evitar litigación por invasión de privacidad: (1) No se meta en la vida privada de las personas a menos que le pidan. (2) Mantenga en secreto los asuntos privados de otras personas. (3) Informe a las personas con antelación cuando usted decide grabar una conversación. Finalmente, (4) consiga permiso antes de publicar una fotografía, cita o grabación. ${ }^{13}$

12 Elena G. de White, El hogar advenstista (Mountain View, CA: Pacific Press Publishing Association, 1959), 156.

13 Taylor. 


\section{Abuso infantil}

El abuso infantil es definido como "el maltrato emocional o sexual de niños" ${ }^{14} \mathrm{El}$ abuso de niños es un problema que ha plagado a las iglesias por décadas. ${ }^{15}$ Negligencia y falta de supervisión es la causa principal para el gran número de juicios sobre acoso infantil en contextos religiosos. ${ }^{16}$ De acuerdo al Instituto de Investigación y Prevención de Acoso Infantil, ${ }^{17}$ la mayoría de los niños son abusados y acosados sexualmente 82 por miembros de su familia o amigos cercanos. Se estima que

14 Princeton University, "child abuse”, WordNet Search-3.1, http:// wordnetweb.princeton.edu/perl/webwn?s=child\%20abuse (consultado: 15 de septiembre, 2013).

15 James D. Berkley, ed., Leadership Handbook of Management and Administration: Practical Insights From a Cross Section of Ministry Leaders (Grand Rapids, Mich.: Baker Books, 1997), 490.

16 Hammar, 772.

17 Véase, Early Diagnosis and Effective Treatment, "Your Opportunity To Save Children", Child Molestation Research \& Prevention Institute, http://www.childmolestationprevention.org/index.html (consultado: 15 de septiembre, 2013). dos de cada tres niñas y uno de cada diez niños son sexualmente abusados antes de los catorce años de edad.

Líderes religiosos han expresado preocupación por la aceptación de miembros de iglesia que han sido enjuiciados o encarcelados por acoso infantil. Un documento preparado por la Asociación de Congregaciones Universalistas Unitarias $(\text { ACUU })^{18}$ prescribe que a una persona con un expediente de ofensas sexuales en contra de niños, no debiera permitírsele acompañar a infantes, trabajar o socializar con ellos y con adolescentes. Ninguna persona con acusaciones sexuales pendientes o con un historial de mala conducta sexual debiera participar en actividades educacionales o religiosas que involucren niños. De acuerdo a la ACUU, el mensaje que debe dársele a los acosadores sexuales es que pueden

18 Unitarian Universalist Association, "Guidelines for Involving Sex Offenders", Unitarian Universalist Association of Congregations, http://www.uua.org/safe/children/offenders/index.shtml (consultado: 15 de septiembre, 2013). 
participar en actividades educacionales y religiosas con adultos, pero que deben comprometerse con la congregación a no entrar en contacto con niños.

Reportando casos de abuso infantil

Los ministros con frecuencia llegan a conocer casos de abuso infantil durante sesiones de consejería o a través de informes que reciben de ciertos miembros de iglesia. Un gran número de gobiernos civiles exigen que los ministros denuncien casos de abuso infantil y sancionen criminalmente a quienes no lo hacen. Existe evidencia de cortes que han rechazado la defensa de algunos ministros que no denunciaron casos de abuso infantil bajo el argumento de tratar el caso "dentro de la congregación". 19

\section{Medidas preventivas}

Las iglesias pueden evadir litigaciones asociadas con abuso infantil implementando un programa riguroso para filtrar a empleados y personas que trabajan con niños. Tanto voluntarios como personas que solicitan trabajar en escuelas y departamentos de niños deben ser cuidadosamente entrevistados, además deben poseer meritorias recomendaciones para trabajar con menores, es decir, su historial y experiencia en el trabajo con niños, deben ser verificados. Cada denominación tiene el deber de actualizar a sus ministros con las leyes sobre abuso infantil que rigen sus territorios.

\section{El alquiler del edificio}

El crecimiento y expansión de nuevas congregaciones y el costo monumental de los edificios de adoración, ha dejado a muchas nuevas congregaciones con la única opción de alquilar salones para sus servicios de adoración. Aunque alquilar una iglesia no le quita a la denominación su estatus de sostén propio ni de exención de impuestos, la congregación puede tener la obligación de pagar impuestos de la ganancia de alquileres si

19 Berkley, ed., 491. 
no tiene deudas pendientes. ${ }^{20}$ Alquilar salones de adoración de otras denominaciones, como alquilar edificios propios a otros grupos religiosos, requiere que se firmen contratos por personas que tengan autoridad ejecutiva en ambas instituciones. Un miembro de iglesia no debe servir como agente para alquilar un edificio donde se sostendrán servicios religiosos de la denominación.

\section{Recomendaciones prácticas}

Para la compra o alquiler de 84 edificios, es necesario recibir asesoría legal. Las Asociaciones Adventistas normalmente cuentan con contratos y abogados que pueden asesorar en la venta y alquiler de propiedades. Sin esta documentación y asesoría,

20 David J. Hardy, "What must our congregation consider before leasing our building?", Evangelical Lutheran Church in America, http://www.elca.org/WhoWe-Are/Our-Three-Expressions/Churchwide-Organization/Office-of-the-Secretary/Congregation-Administration/ Legal-Issues-for-Congregations/Questions-About-Buildings-and-Property/ What-must-our-congregation-consider-before-leasing-our-building.aspx (consultado: 14 de mayo, 2009). ninguna persona debiera aventurarse a alquilar o comprar edificios para la iglesia. Debe aclararse que ciertas regulaciones civiles, en ciertas comunidades, no permiten reuniones religiosas dentro de sus límites, de manera que comprar o alquilar un edificio con esos fines, es una infracción a las reglas que, como iglesia, debemos evitar.

Contratos para alquilar edificios a otras denominaciones deben incluir una cláusula que exonere a la iglesia de daños y perjuicios causados por accidentes que dañen a personas que alquilan nuestros edificios. Es crucial que las personas que alquilan la iglesia provean una copia del seguro de accidente que cubra a sus miembros; además, debe hacerse un horario maestro de reuniones para evitar reuniones conflictivas.

\section{Asuntos legales asociados con excursiones}

"Responsabilidad" es una de las palabras legales más importantes en la administración 
de iglesias. En el contexto de la iglesia, esta palabra significa protección y cuidado de los feligreses. Negligencia en asegurar la protección y cuidado de los creyentes pone a la institución en peligro de juicio. La responsabilidad con los miembros de iglesia se extiende a excursiones dentro y fuera del territorio de la Asociación o Misión. La iglesia no debiera contratar choferes sin experiencia o con mal historial en la conducción de vehículos. Accidentes causados por tales choferes pueden ser acreditados a la institución por no contratar a conductores sin las cualificaciones necesarias.

La iglesia debe hacer todo lo posible para proteger a sus constituyentes, lo que incluye la adquisición de una póliza de "seguro de responsabilidad civil" ${ }^{21}$ para proteger el cuidado y bienestar de sus feligreses, en caso de accidente durante actividades organizadas por la iglesia. El seguro de compensación y cuidado médico para tra-

21 Conocido en inglés como "Liability Insurance". bajadores y miembros de iglesia debe estar al día con la póliza de la denominación. ${ }^{22}$

\section{Recomendaciones prácticas}

Las siguientes recomendaciones proveen orientación en caso de que alguna persona se lesione fuera de las premisas de la iglesia, durante una actividad debidamente aprobada por el comité o junta de la iglesia: (1) Antes de salir a la expedición con el grupo, lleve la documentación del seguro de la iglesia. (2) En caso de accidente, evite comentarios que puedan comprometer a alguna persona $\mathrm{O}$ entidad -e.g. "las escaleras han estado dañadas por más de dos años" o "el chofer no tenía experiencia”-. (3) Haga arreglos para que la persona herida sea llevada a un hospital y reciba atención médica. (4) Haga un informe del accidente que incluya detalles tales como el día, hora y lugar del accidente; tipo

22 Bruce P. Powers, ed., Church Administration Handbook: A Revised and Completely Updated Edition (Nashville: Broadman \& Holman Publishers Group, 2008), 223. 
de actividad, naturaleza de la lesión y otros detalles relevantes. (5) Evite actividades peligrosas como canotaje en ríos con rápidos, el paracaidismo o deportes extremos. (6) Finalmente, tome un voto del comité de la iglesia para aprobar la salida. Recuerde que para que el seguro de la iglesia cubra accidentes de actividades fuera de la congregación, es necesario que la actividad sea autorizada por el comité de la iglesia local.

\section{Conducta sexual} 86 inapropiada

La conducta sexual inapropiada se manifiesta de muchas formas y es responsable de muchos litigios en contra de la Iglesia. Los ministros deben ejercer mucho cuidado y tacto al conversar o aconsejar a miembros adultos del sexo opuesto, especialmente cuando las sesiones revelan asuntos íntimos y explícitos. Esposos celosos o en desacuerdo con el consejo del pastor, pueden acusarlo de alienación de la esposa o de enfriar su afecto conyugal. Tales acusaciones, aunque sean falsas, pueden generar escándalos con el potencial de dañar la reputación del ministro y de la iglesia. Taylor divide la conducta sexual inapropiada en cuatro áreas legales: (1) abuso sexual infantil; (2) conducta sexual inapropiada con adultos; (3) acoso sexual en el trabajo y (4) la responsabilidad de la iglesia con la conducta sexual inapropiada de sus empleados. ${ }^{23}$ Shaughnessy ${ }^{24}$ afirma que la conducta sexual inapropiada puede incluir situaciones y acciones inocentes como una palmadita amigable en el hombro de una persona o acciones deliberadas, tales como la insinuación de contacto sexual.

La Universidad Estatal de Virginia, EE.UU., define la conducta sexual inapropiada como el contacto sexual sin consentimiento por parte de un conocido o un extraño. Comportamientos sexuales inapropiados incluyen tocar in-

23 Taylor, 79.

24 Shaughnessy, 55. 
tencionalmente a otra persona sin su consentimiento, intento de violación, sodomía ${ }^{25}$ sin consentimiento o penetración sexual sin consentimiento. Acciones sin consentimiento son aquellas a las que se consiente por amenaza, fuerza, intimidación o aprovechamiento de la inhabilidad física o mental de la víctima; tal incapacidad puede ser inducida por alcohol, drogas o estupefacientes. ${ }^{26}$ Aunque el agresor es el responsable de un abuso sexual, en el contexto de la iglesia, un gran número de leyes civiles responsabiliza a la congregación por negligencia en la protección de sus miembros. ${ }^{27}$

25 Sexo oral o anal.

26 University of Maryland. Retrieved September 16, 2013, véase, https:// my.umary.edu/ics/ClientConfig/CustomContent/policies/Sexual_Misconduct_ Policy.pdf

27 Una corte en Colorado, EE.UU. remuneró a una víctima sexual con más de un millón de dólares, declarando que la iglesia no había indagado acerca del historial del violador, que era el pastor de la iglesia. En otro caso en Ohio, EE. UU., una mujer reclamó que había sido violada por el pastor durante una sesión de consejería y ganó un juicio contra la iglesia por

\section{Licencia de consejería}

Muchas iglesias han sido llevadas a juicio por la conducta inapropiada de ministros durante sesiones de consejería. Ministros que no tienen licencia de consejería no debieran aventurarse a dar consejería matrimonial y debieran referir los pacientes a profesionales capacitados para ese fin. ${ }^{28}$

El acoso sexual es considerado como una forma de discriminación ${ }^{29}$ y es asociado con insinuaciones verbales y conducta física que insinúan favor sexual. ${ }^{30}$ En el contexto del trabajo, el acosador sexual tiende a ser persistente y usa comentarios degradantes, amenazantes

no haber ejercido cuidado al emplear a un ministro con tendencias sexuales inapropiadas. Véase, Hagglund y Weimer, 26, 27.

28 Powers, ed., 223.

29 Esta forma de discriminación es prohibida en los EE.UU. por la ley: Title VII of the Civil Rights Act of 1964. Por otro lado, el acoso sexual es una violación señalada en la Sección 703 del Título VII. Véase, Hammar, 545.

30 Véase, Legal Dictionary, "Sexual Harassment", The Free Dictionary, http:// legal-dictionary.thefreedictionary.com/ sexual+harassment (consultado: 15 de setiembre, 2013). 
y sexistas. La víctima sabe que de no ceder a las insinuaciones sexuales del agresor, puede perder beneficios y posiblemente el empleo. ${ }^{31}$ Otras conductas asociadas con el acoso sexual incluyen el abuso verbal repetido y el uso de gestos obscenos. ${ }^{32}$

\section{La prevención de conducta sexual inapropiada}

Hay varias cosas que la iglesia puede hacer para disminuir incidentes de conducta sexual inapropiada. ${ }^{33}$ (1) Evitar consejería pastoral uno-a-uno. La adopción 88 de una regla que excluya la consejería de una persona del sexo opuesto sin la presencia de una tercera persona tiene el potencial de reducir considerablemente la posibilidad de acusaciones de acoso sexual. (2) Restringir la consejería fuera de las instalaciones de la iglesia con especificaciones que incluyan la presencia de una tercera persona y la mención del sitio donde tendrá

31 Véase, Law.com.s.v. "Sexual Harrasment"

32 Taylor, 92.

33 Este consejo es adaptado de Hagglund y Weimer (1998) y Hammar (2000). lugar la sesión. (3) Confinar la consejería a conversaciones telefónicas que no duren más de una hora. (4) Educar a los líderes de la iglesia, de todos los niveles, en los peligros y obligaciones asociados con la conducta sexual inapropiada. Los líderes de la iglesia deben ser alertados de las acciones y situaciones que conducen a ese tipo de conducta. (5) La iglesia debe investigar expeditivamente cualquier reclamo o rumor de abuso sexual. $\mathrm{Si}$ el abuso sexual es confirmado, el empleado debería ser despedido inmediatamente.

\section{La prevención del acoso sexual}

La mayoría de las denominaciones tienen pólizas establecidas acerca del acoso sexual. ${ }^{34} \mathrm{Ta}$ les pólizas deben estipular con claridad que el acoso sexual no es tolerado y que forma la base para perder el trabajo. Las pólizas también deben: (1) establecer con claridad un proceso para archivar una queja de acoso con

34 La póliza de la Iglesia Adventista en contra del acoso sexual está disponible en http://www.adventist.org/beliefs/guidelines/main_guide6.html 
la administración; (2) animar a las víctimas a que reporten incidentes de acoso; (3) asegurar a las víctimas a que las quejas de acoso son investigadas inmediatamente; (4) asegurar a las víctimas que no recibirán ningún tipo de retaliación por presentar una queja en contra de un superior; (5) establecer medidas disciplinarias en contra de los agresores que violan las pólizas de la denominación y (6) asegurar la confidencialidad a las víctimas. ${ }^{35}$

\section{Infracción de los derechos de autor}

Una de las áreas violadas con más frecuencia por miembros y líderes de la iglesia tiene que ver con los derechos de autor. Las leyes que regulan los derechos de autor no son bien compren-

35 Más consejos sobre el tema son sugeridos por Hammar, véase: "Tips on reducing legal risk", http:// ag.org/top/Legal_Matters/index_legal_liability_detail.cfm?targetBay $=80$ 5ba5b6-a4c0-4d22-8ec3-502987e6b7 ee $\&$ ModID $=2 \&$ Process $=$ DisplayArti cle\&RSS_RSSContentID $=15287 \&$ RSS OriginatingChannelID $=1158 \&$ RSS_OriginatingRSSFeedID $=4503 \&$ RSS_Source $=$ didas por los administradores y miembros de iglesia. Estas leyes otorgan a los músicos y autores los derechos únicos para copiar, publicar y distribuir sus obras escritas y musicales. Estas obras pueden incluir libros, grabaciones, estudios bíblicos, artículos, programas de computadora, películas y materiales audiovisuales. ${ }^{36}$

\section{El uso inapropiado del nombre de la iglesia}

La mayoría de las iglesias, incluyendo la Iglesia Adventista del Séptimo Día, tiene marca registrada de sus nombres, los cuales no pueden utilizarse sin un consentimiento escrito. Por ejemplo, la palabra "Adventista", tiene derechos registrados, de manera que ninguna otra organización o entidad la puede usar sin permiso escrito. Ser miembro fiel de la iglesia y el apoyarla financieramente, no

36 Para que una obra sea registrada con derecho de autor, debe ser original y ser presentada en forma tangible. Las ideas, títulos, símbolos y descubrimientos no pueden registrarse con derecho de autor. Véase, Berkley, ed., 502. 
otorga a sus miembros ningún derecho para utilizar el nombre de la iglesia con fines personales, aunque esos fines sean de carácter religioso, como la formación de un movimiento de sostén propio.

\section{Recomendaciones prácticas y medidas preventivas}

Los miembros de iglesia deben ser los ojos "de la denominación" para ver si hay personas o entidades que usan nuestro nombre sin permiso. Las iglesias deben instruir a sus miembros y músicos acerca de las leyes que regulan los derechos de cada autor. Ningún tipo de material escrito o musical debe duplicarse sin permiso escrito del autor. La violación de esta ley puede conducir a juicios legales. Es importante que el ministro conozca las leyes de autor vigentes en el país donde trabaja. ${ }^{37}$

37 La Organización Mundial de la Propiedad Intelectual (OMPI) ha publicado un tratado sobre derechos de autor internacionalmente aceptado. Véase, http:// www.wipo.int/treaties/es/ip/wct/index. html. Cada país tiene una entidad en la
La iglesia no debe hacer copias de ninguna publicación con derecho de autor. Algunas excepciones son permitidas con fines de criticismo, evaluación, reportaje con fines noticieros, fines académicas, ${ }^{38} \mathrm{o}$ para fines de investigación..$^{39}$ La organización Christian Licensing International se especializa en obtener permiso para publicaciones musicales. ${ }^{40}$

\section{Conclusión}

Los líderes de instituciones

que se puede registrar el derecho de autor de obras personales. En los EE.UU., las leyes de derecho de autor pueden obtenerse escribiendo a: U.S. Copyright Office, 101 Independence Ave., S.E., Washington, DC 20559-6000, o visitando su portal cibernético: http://www.copyright.gov/eco/index. html.

38 Por ejemplo, la fotocopia de materiales para el uso en el aula de clase, sin fines de lucro financiero.

39 Powers, ed., 229.

40 Esta organización cobra una cuota anual por sus servicios. Información detallada acerca de esta organización puede obtenerse en su página web, http://www. ccli.com/. Información por correo regular puede obtenerse escribiendo a la siguiente dirección-CCLI, 17201 N.E. Sacramento Street, Portland, OR 97230. 
religiosas deben mantenerse en sintonía con las leyes más recientes que aseguran el bienestar físico y emocional de los miembros de iglesia de todas las edades. Educación continua y actualizada, tanto de los ministros como de los miembros de iglesia puede reducir considerablemente el riesgo de juicios costosos y dolorosos.

\section{Bibliografía recomendada}

Varios libros se han escrito acerca de los desafíos legales que el pastor enfrenta en el ministerio, con el fin de proteger a los miembros de iglesia y prevenir juicios en contra de la organización. Se recomienda las siguientes publicaciones entre las que abordan el tema:

Taylor, Thomas F. Seven Deadly Lawsuits: How Ministers Can Avoid Litigation and Regulation. Nashville, TN: Abingdon Press, 1996.

Hagglund, Clarance E. y Britton D. Weimer. Stay Out of Court and Stay in Ministry. Lima, OH: CSS Publishing Company, 1998.
Shaughnessy, Mary Angela. Ministry and the Law: What you Need to know. New York: Paulist Press, 1998.

Hammar, Richard R. Pastor, church $\mathcal{E}$ law. 4ta ed. Matthews, NC: Christian Ministry Resources, 2008.

Este último volumen es usado como referencia para asuntos legales por abogados de instituciones religiosas.

\section{Bibliografía general}

Berkley, James D., ed. Leadership Handbook of Management and Administration: Practical Insights From a Cross Section of Ministry Leaders Grand Rapids, Mich.: Baker Books, 1997.

Gumper, Lindell L. Legal Issues in the Practice of Ministry. Birmingham, MI

Psychological Studies and Consultation Program, 1981.

Powers, Bruce P., ed. Church Administration Handbook: A Revised and Completely Updated Edition Nashville: Broadman \& Holman Publishers Group, 2008.

Real Academia Española, Diccionario de la lengua española: Real Academia Española. Madrid: Espasa Calpe, 2003.

White, Elena G. de. El hogar advenstista. Mountain View, CA: Pacific Press Publishing Association, 1959. 